\title{
SIII-2b
}

\section{Vegetation insulation screen as a passive cooling system in hot humid climate: heat and mass exchanges}

\author{
Hodo-Abalo Samah'1, 2, Magolmèèna Banna², and Belkacem Zeghmati ${ }^{3}$
}

\section{Abstract}

Planted roofs are passive cooling techniques that reduce the thermal load of buildings. In this paper, a dynamic mathematical model based on time average Navier-Stokes equations for a planted roof in hot humid climates has been developed for evaluating the cooling potential.Transfer equations are solved using a finite difference scheme and Thomas algorithm. The model was applied for the simulation of the planted roof in togolese climate conditions. Results showed that, evapotranspiration and Solar Heat gain Factor are functions of the Leaf Area Index which is the most important parameter when considering the foliage material. It is clearly proved that the foliage density and hence the vegetable canopy type selection greatly influence the thermal efficiency of the bioclimatic insulation screen. It was found that a larger Leaf Area Index reduces the solar flux penetration and increases evapotranspiration which is an important parameter when considering surrounding microclimate formation.

Keywords - Planted roof; Sensible heat; Latent heat; Evapotranspiration; Solar Heat gains Factor

\section{Introduction}

Metallic and concrete roofs are very common in buildings in most of the developing countries particularly in tropical regions. The temperatures inside such buildings are far beyond the comfortable conditions in these regions. The direct cooling devices are electrically operated and are not feasible due to unreliable supply of electricity. In such a situation, passive cooling techniques are very appropriate to provide comfort. Passive cooling of buildings can be defined in several ways [1, 2, 3]. One way is to consider any treatment of the building which reduces its cooling load, such as solar control and minimizing internal heat gains. Studies in the literature show that about 30 to $50 \%$ of the heat gains inside a building are brought about by roof elements [1]. In hot-humid climates, some methods or techniques are better adapted to improve thermal comfort in buildings. Particularly, the use of thermal insulation screens (ground layer, extruded or moulded polystyrene plates, plaster false ceiling, vegetation canopy) underneath and above the concrete flagstone roof reduces the heat load in a building [4, 5]. The insulation screens are integrated beneath reinforced concrete flagstone as an addi-

\footnotetext{
${ }^{1}$ Faculté des Sciences et Techniques (FaST), Université de Kara, Kara -TOGO

${ }^{2}$ Laboratoire sur l'Energie solaire LES/GPTE, Université de Lomé, Lomé -TOGO

${ }^{3}$ Laboratoire de Mathématique et Physique des Systèmes, Université de Perpignan via Domitia, Perpignan, France
} 
tional insulation sublayer. The aim of the vegetation surface on roof top design concept is to reduce the heat gain and minimize the cooling load for mechanical air-conditioning. The field study shows that the passive cooling of planted roofs is recognized as one of the effective energy saving means in buildings in warm-humid climates and reduces the indoor air temperature within comfort range. It influences the use of the electrical cooler and air conditioning of the indoor space. Therefore, it can reduce the energy demand of the building which saves a lot of money for the daily electricity bill of urban residents [6]. The green roof plants generate oxygen and provide worth for air pollution control and soil erosion. Green roofs reduce the carbon level that controls global warming. Planted roofs present a very effective and positive impact on urban climate and microclimate as well as on the indoor air temperature of buildings beneath them. In closed spaces with planted roofs, the air temperature beneath the plants during sunny times is lower than that of the air above. For their biological functions such as photosynthesis, respiration, transpiration and evaporation, foliage materials absorb a significant proportion of solar radiation and contribute to reduce heat gains inside a building in the highest solar irradiation regions [7]. With rapid urbanization, there has been high growth in population and building construction in cities. A high concentration of buildings actually raises many environmental issues, such as the Urban Heat Island effect. The Urban Heat Island effect started mainly because of the loss of green areas in the urban environment. Plants when strategically placed around roofs, can be considered a complement of urban greens. These provide visual enhancement, noise control, storm water management, improved water run-off quality, improved urban air quality, extension of roof life, reduction of the urban heat island effect, architectural design and biodiversity. This natural solution also contributes to the thermal benefits in buildings and their surrounding environments. Greenery placed around buildings serves to reduce the surface temperature through direct shading of hard surfaces as well as cool the ambient air through absorbing solar heat for transpiration and photosynthesis. The shaded surfaces also emit less long-wave radiation due to lower surface temperature. All these contribute to lowering energy consumption for cooling and mitigating Urban Heat Island effect in the urban environment. The present study aims mainly to analyse heat and mass exchanges in vegetation screen and its effects on internal heat gains of a building. Important parameters which affect the surrounding environments such as evapotranspiration have been analysed as well as sensible and latent heats fluxes. To analyse heat and mass transfer in vegetation screen insulation system, useful parameters such as Nusselt and Sherwood numbers are the main control variables evaluated.

\section{Description of approach and techniques}

The green roof system studied is composed of four interacting subsystems: atmospheric environment above the canopy, the vegetation canopy, the substrate or growing medium and the flagstone as a classic roof. The physical model is shown in Figure 1. Parameters defining the outdoor conditions are the solar radiation flux, the ambient air humidity and temperature, the wind speed and direction. The roof is considered large enough and assumed horizontally homogeneous. Heat and mass fluxes are assumed to be mainly vertical so that one-dimensional model is used to describe the thermal behaviour in the roof components. A reference is chosen at the ground level at soil-canopy interface and the abscissas are counted positively in the sense of plant growth (figure 1). The canopy is composed of the leaves and the air within the leaf cover. It is a complex system of sources and sinks of heat and mass such that an exact 
description of its physical behaviour is almost impossible. While attempting to figure out a simpler model of a canopy, one is faced with two types of problems. The first is the inherent spatial complexity and dishomogeneity of the foliage. This implies that for an accurate description, the number of simultaneous equations to be solved could be five times higher than the number of leaves in the canopy.

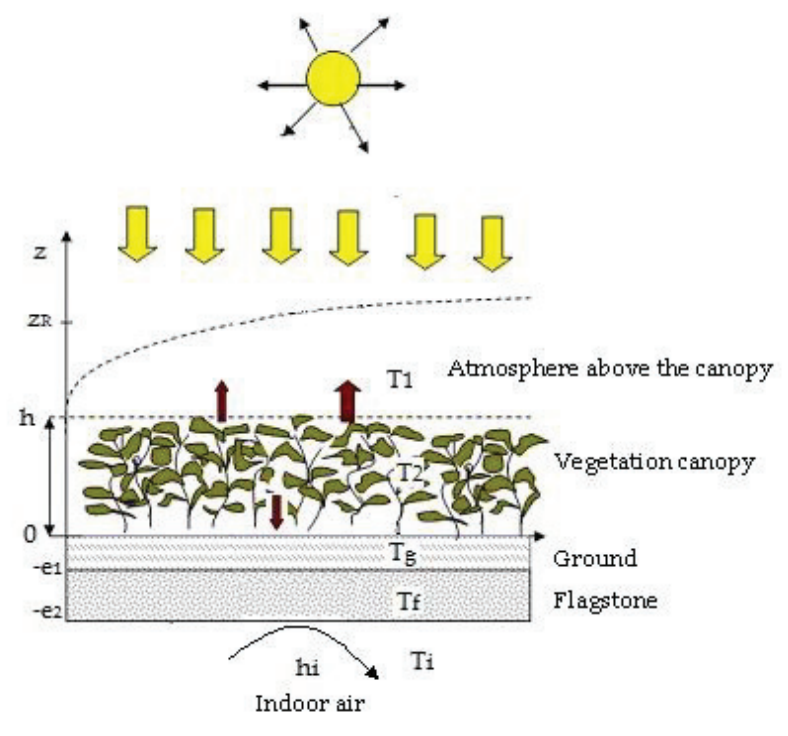

Figure 1: Sketch of a green roof

The second is the turbulent nature of the air stream within a canopy. Its consequence is that the direction and magnitude of the fluxes of energy and mass vary at any moment and cannot be exactly predicted. In spite of this, in much of the literature concerned with the coupling of plants with their environment, heat and mass transfer to and from a canopy are described as vertical fluxes along a concentration gradient across some typical resistances. For a given vegetation canopy, the leaf and the stalk constitute obstacles to airflow and are characterised by the Leaf Area Density $\operatorname{LAD}(\mathrm{z})$. This scalar is assumed to be a vertical distribution function. In leaf gas exchange studies, the variable employed to characterise the atmospheric water stress was the leaf-to-air saturation deficit $Y(z)$. This scalar is defined as the difference between dry and wet air bulk temperatures: $Y(z)=T(z)-\operatorname{Tr}(z)$.

The transpiration rate depends on the bulk stomatal coefficient $\alpha(z)$ which plays the same role as bulk stomatal resistance $r(z)$ of the plants reported by most authors. The coefficient $\alpha$ is equal to 0 for the dry foliage plants and 1 for the wet foliage. When the vegetation canopy is very dry, evaporation comes under the physiological control of the plant, as it has to pass through the stoma. The bulk stomatal resistance of the canopy is expressed using the Noilhand and Planton correlation [8], the factors considered here are a solar radiation factor $\mathrm{f}_{1}$, a water stress factor $f_{2}$, a factor related to atmosphere pressure deficit $f_{3}$ and an air temperature dependence factor $f_{4}$. These different factors $f_{i}$ are detailed in the reference [9].

Transfer equations with appropriate boundary conditions are solved numerically using an implicit finite difference scheme and Thomas algorithm. The diffusion terms are approximated by the central finite difference scheme and the implicit procedure to discretize the temporal derivatives is retained. For a given temperature at substrate-canopy and canopy-atmosphere interfaces and under initial conditions, water vapour, heat and momentum transfer equations are 
solved in each zone. The following time and spatial steps $\Delta \mathrm{t}=10 \mathrm{~s}$ and $\Delta \mathrm{x}=0.005 \mathrm{~m}$ are respectively retained. Successive iterations were applied and the solution is considered satisfactory when the following convergence criterion is verified for each time:

$$
\left|\left(\phi_{i, j}^{k+1}-\phi_{i, j}^{k}\right) / \phi_{i, j}^{k}\right| \leq 10^{-5}
$$

$\phi$ can be the temperature $\mathrm{T}$ or the water vapour density $\mathrm{C}$

\section{Results and discussion}

The daily ambient temperature and solar radiation are assumed to have a sinusoidal variation during a day. For a given ambient temperature and relative humidity, the dew points are deduced from Mollier's psychometric chart. The saturation deficit scalars of the air as well as the water vapour density at the reference height are calculated. Particular attention is paid to characteristic fluxes such as evapotranspiration, sensible and latent heats fluxes. The local heat gain across the planted roof is evaluated in terms of solar heat gain factor. For a given value of wind speed in greenery, effects of solar radiation and canopy structure (Leaf Area Index LAl) on evapotranspiration, sensible and latent heats fluxes are illustrated in figures and analysed. The determination of water vaporization and its transfer to substomal cavities according to climatic characteristics, canopy structure and stomatal regulation is an essential point in the understanding and interpretation of evapotranspiration. Indeed, hourly evapotranspiration is obtained by summing hourly values of local latent-heat flux from different layers within the canopy and added to the hourly value of evaporation at substrate's level. The evolution of evapotranspiration density as a function of the incident solar flux density is illustrated in Figures 2. The evapotranspiration varies linearly as a function of solar radiation density. The characteristic result of evapotranspiration for different values of Leaf Area Index LAl show how evapotranspiration evolves over a sunny day. An increase in LAl value resulting from vegetation canopy density increases the evapotranspiration value. It's clear that evapotranspiration is more important when the foliage is sufficiently dense.

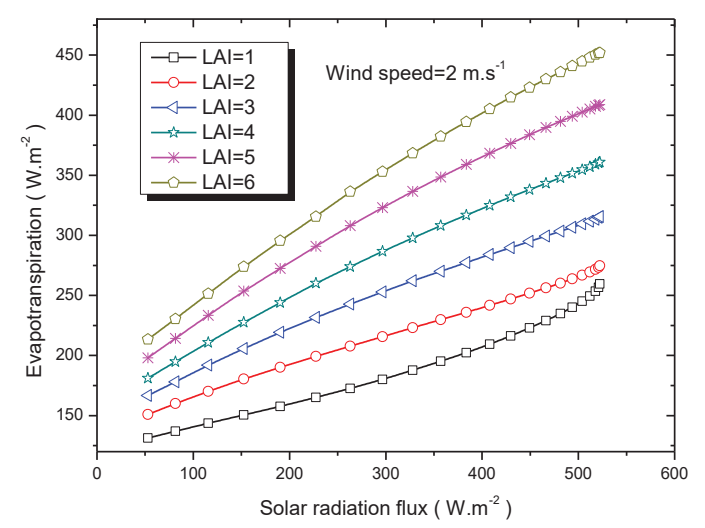

Figure 2: Evapotranspiration as a function of solar radiation density

Net radiation in the canopy is a function of $\mathrm{LAl}(\mathrm{z})$; therefore, for a high value of LAl, transpiration heat flux is more important and heat removal involves a decreasing of temperature within the canopy. In fact, according to the intensity of the latent heat flux which depends directly up- 
on biological regulation, each part of the plants becomes a local source of heat for weak evaporation or local sink of heat for strong evapotranspiration.

$$
\mathrm{LAI}=2
$$
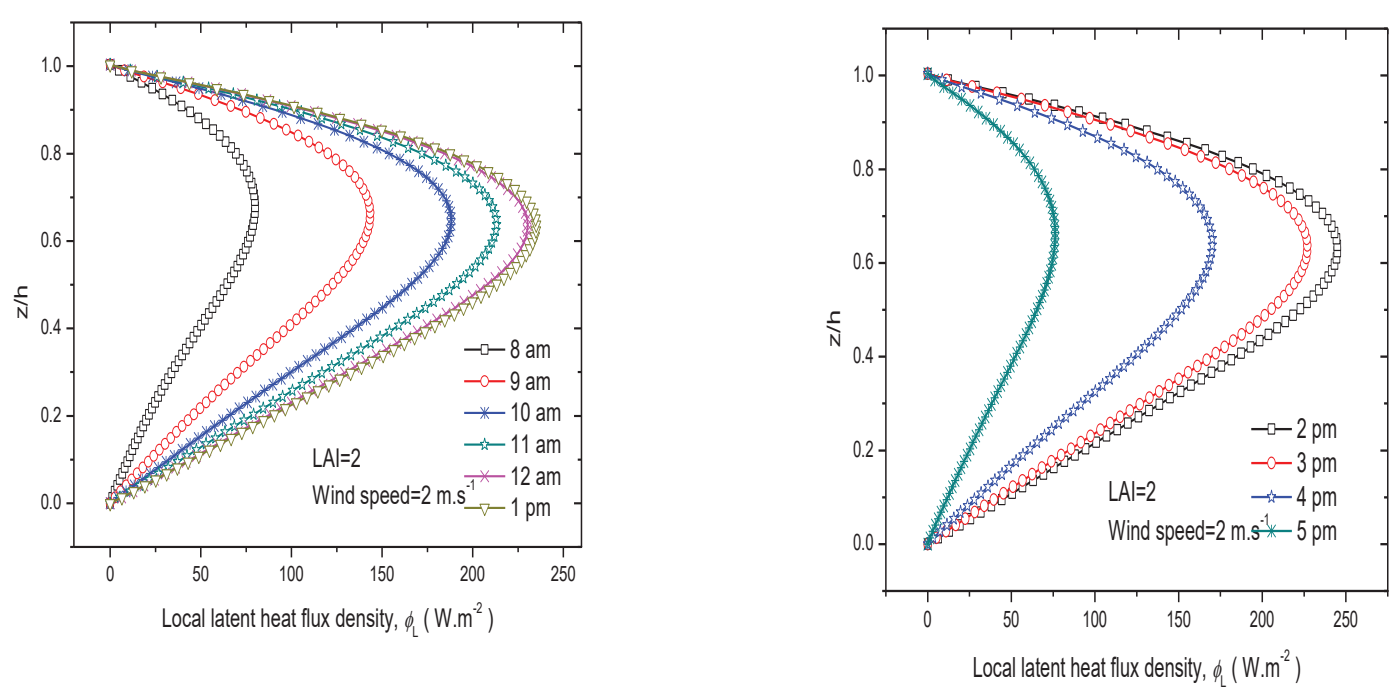

$\mathrm{LAI}=6$
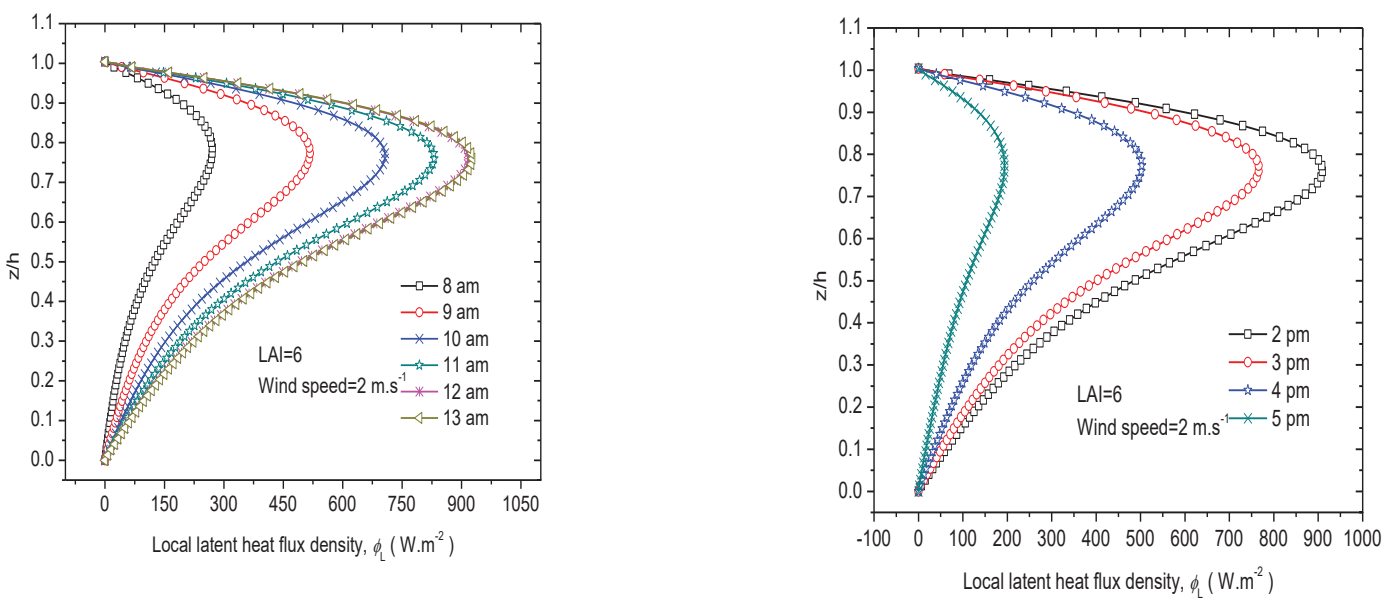

Figure 3: Local latent heat flux density profiles within the canopy

In figures 3 and 4 are illustrated the variation of the sensible and latent heat fluxes at different hours of a day. As expected, according to the leaf density area distribution, maximum transpiration source occurs at the mid-height $h / 2$ of the vegetation canopy. A striking decrease in transpiration is observed in the upper and lower parts of the canopy. The distribution of the leaf area density plays a very important role in heat and mass exchange in the canopy. The symmetrical distribution adopted leads to a local latent heat flux density that decreases from mid-canopy height to the soil surface under the canopy as shown in Figure 3. This decrease is more or less modified by the value of stomatal resistance that can reduce, by stomatal closure, water loss, and energy loss by sensible heat flux and the increase in surface temperatures. A strong stomatal regulation in the upper part of the canopy could lead to a maximum flux 
density of local latent heat. A specific arrangement of the orientation of the sheets with a minimum captation of radiation in the upper part of the canopy may also lower the maximum flux density of local latent heat. For low foliar coverage, local latent heat flux density is not high enough since the maximum obtained around 2 p.m is about $250 \mathrm{~W} . \mathrm{m}^{-2}$ while in the case of a dense canopy, this flux density can reach about $900 \mathrm{~W} \cdot \mathrm{m}^{-2}$.

\section{$\mathrm{LAI}=2$}

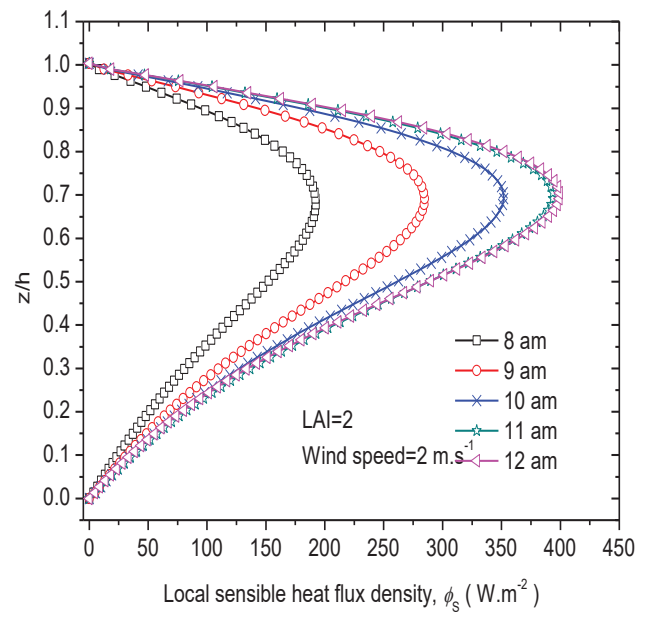

\section{$\mathrm{LAI}=6$}

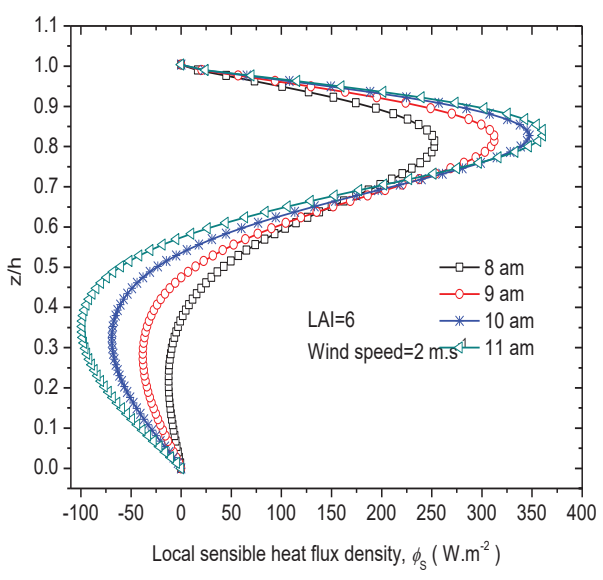

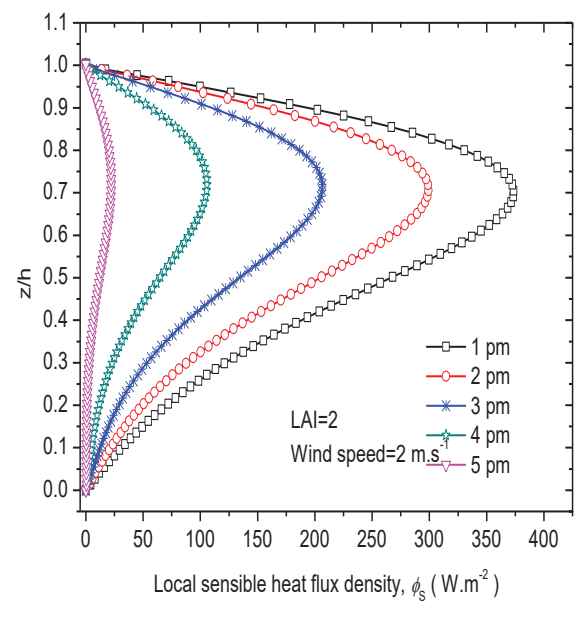

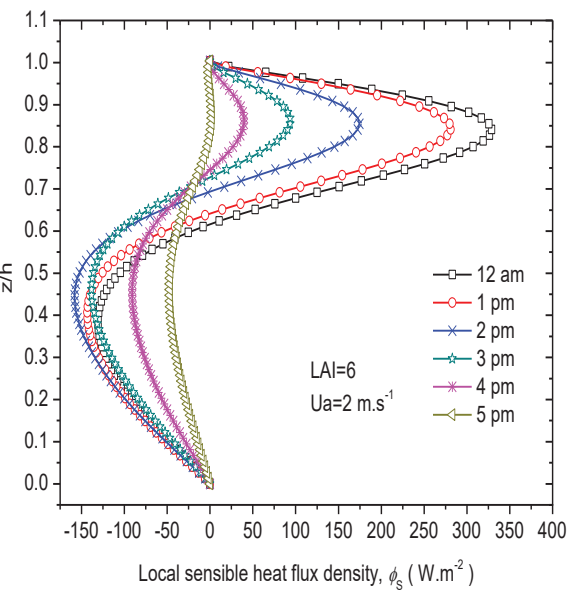

Figure 4: Local sensible heat flux density profiles within the canopy

The local density of sensible heat flux evolves in more complex manner with mostly positive values in the upper parts of the canopy, sometimes turning negative in the lower parts as it's shown in figure 4. Heat flux exchange from the canopy to the atmosphere explained the positive values of sensible heat flux while negative values obtained mostly for large values of LAI are explained by the diffusion of the accumulated heat in canopy towards the colder the substrate. Figure 5 illustrates the temperature profiles of the substrate surface for different LAI values. This figure shows that an increase in LAl causes a decrease in temperatures. A fairly dense vegetation cover considerably reduces the growing medium temperature. This is explained by the fact that the net radiation is strongly mitigated by the leaf material and by the 
activity of the stomata which are more numerous. In general, the growing medium surface temperature is sensitive to climatic factors, the type of vegetation cover is highly dependent on the state of drought of this layer.

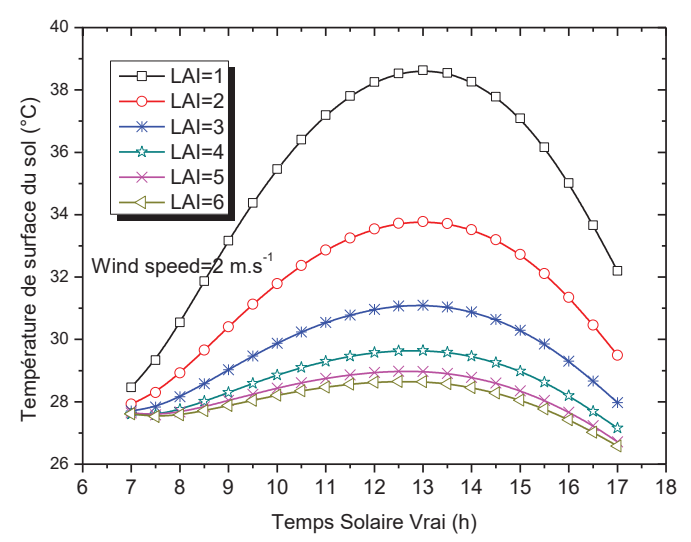

Figure 5: Evolution of surface temperature of the substrate

Phenomena of heat and mass exchanges modelling are described in terms of Nusselt and Sherwood numbers. Figure 6 (a) and (b) presents respectively the variations of the Nusselt and Sherwood numbers as a function of the solar radiation density for differents values of LAl. The both two transfer numbers evolve linearly as a function of solar radiation. For a given LAl, the intensity of heat (Figure $6(\mathrm{a})$ ) and water vapor (Figure 6 (b)) transfer at the vegetationatmosphere interface intensify when increasing solar radiation density which in fact controls the stomata regulation. The profiles of transfer nummbers increase with the value of LAl. For high values of LAl, transpiration flux increases significantly and exchanges of heat and water vapor between the vegetation and the atmosphere are very important as shown by the variations of the Nusselt and Sherwood numbers.
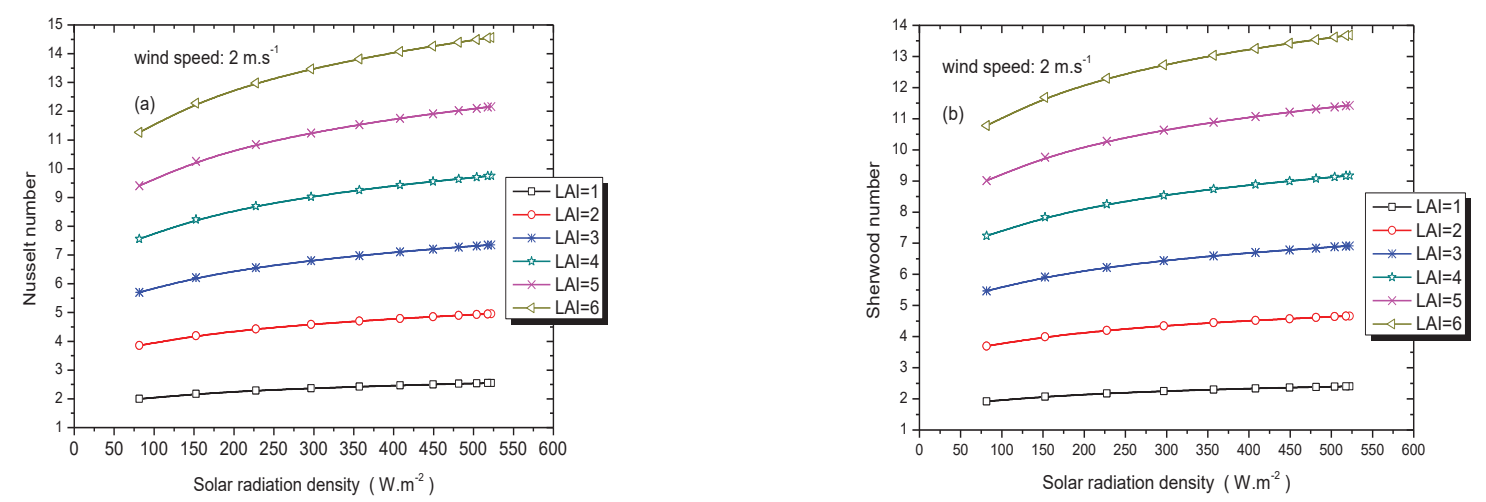

Figure 6: Nusselt (a) and Sherwood (b) numbers as a function of solar radiation density

When considering the degree to which solar gain is transmitted by the fabric of the building, it is helpful to use the concept of solar heat gain factor. This is a useful parameter defined by Koenigsberger [10] as the heat flow rate through the construction due to solar radiation expressed as a fraction of the incident solar radiation. In the present study, the solar heat gains factor of the green roof is defined as the ratio of transmitted solar energy into the interior of the building to incident solar energy. This factor has been calculated for the planted roof and for 
the roof without vegetation canopy. Figure 7 clearly indicates that, the vegetation canopy significantly reduces the heat gain in the building. Indeed, for a given wind speed of $1.5 \mathrm{~m} . \mathrm{s}^{-1}$ and without greenery on the roof top, the solar heat gain factor can reach a maximum value of $45 \%$ against $15 \%$ for a roof with vegetation canopy ( $\mathrm{LAI}=3$ ).

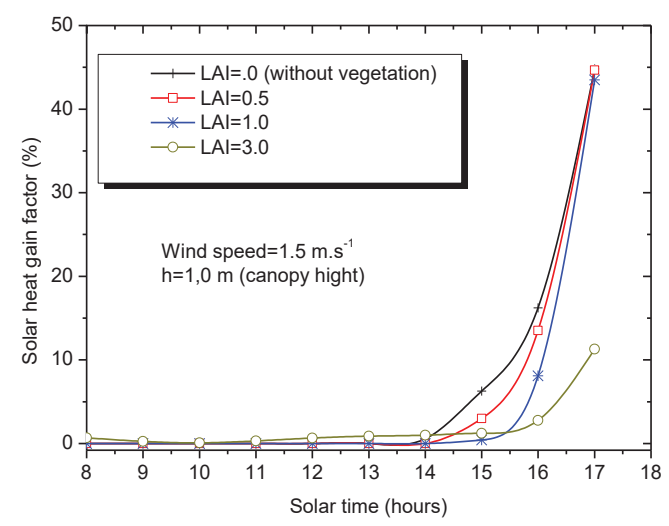

Figure 7: Hourly evolution of solar heat gain factor

\section{Conclusions / Perspectives}

A dynamic mathematical model based on time average Navier-Stokes equations for a planted roof in hot humid climates has been developed. The effectiveness of vegetation screen as a passive cooling technique is examined theoretically and compared with a clas sical roof in hot-humid climate conditions. The results showed that solar heat gain factor can be can reduced to $15 \%$ for the planted roof against $45 \%$ for for bare roof. It is clearer, that cooling load in buildings can be reduced by the application of such bioclimatic screen design. Evapotranspiration and Solar Heat gain Factor are functions of Leaf Area Index which is the most important parameter when considering the foliage density. It is clearly proved that the foliage density and hence the vegetation canopy type selection greatly influence the thermal efficiency of the bioclimatic insulation screen. It was found that a larger Leaf Area Index can reduce the solar flux penetration and increases evapotranspiration which is an important parameter when considering surrounding microclimate formation.

Before, adoption of such a technique in hot humid climate, studies must be conducted not only on plants species selection suitable with minimum maintenance but also on the resistance of species selected to drought and their water consumption for possible application to the arid regions.

\section{References}

[1] N. M. Nahar, "Studies on solar passive cooling techniques for arid areas", Energy conversion and Management, Vol. 40, pp. 89-95, 1999.

[2] Runsheng Tang \& Y. Etzion, "On thermal performance of an improved roof pond for cooling Buildings", Building and Environment, Vol. 39, pp. 201-209, 2004

[3] Dilip Jain, "Modeling of solar passive techniques for roof cooling in arid regions", Building and Environment, vol. 41, pp. 277-287, 2006 
[4] H. Abalo Samah \& M. Banna, "Performance analysis of Thermal Insulation Screens used for Classic Roofs in hot-humid Tropics", International Energy Journal, Vol. 10, pp. 255266, 2009.

[5] S Elena Palomo Del Barrio, "Analysis of the green roofs cooling potential in buildings", Energy and Buildings, Vol. 27, pp. 179-193, 1998.

[6] Rumana Rashid, "Financial and Environmental Benefit of Pot Plants' Green Roof in Residential Building in Bangladesh" World Journal of Management, Vol.2, №2, Pp. $45-50$, 2010.

[7] Kumar R. \& S.C. Kaushik, "Performance evaluation of green roof and shading for thermal protection of buildings", Building and Environment, Vol. 40, pp. 1505-1511, 2005.

[8] Noilham \& J.Nad PI., "A simple parameterization of land surface processes for meteorological model”, Mon. Weather Rev., Vol. 117, pp. 536-549, 1989.

[9] D L. Seen, "An approach to couple vegetation functioning and soil-vegetationatmosphere-transfer models for semiarid grasslands during the HAPEX-Sahel experiment", Agricultural and Forest metrology, Vol. 83, pp. 49-74, 1997.

[10] Koenigsberger O. H, Ingersoll T G, Mayhew A \& Szokolay S V., "Manuel of tropical housing and building design“, Longman. London, 1973. 